\title{
CICLO BIOLOGICO Y ESTABLECIMIENTO DE UNA COLONIA DE ANOPHELES PSEUDOPUNCTIPENNIS THEOBALD 1901, VARIEDAD BIFOLIATA (DIFTERA: CULICIDAE) EN CALI, COLOMBIA (1)
}

\author{
LYDA RAMIREZ*, PATRICIA MEDINA*, PAULINA FAJARDO**, RANULFO GONZALEZ*** \\ Anopheles pseudopunctipennis, variedad bifoliata, fue colonizado por \\ cinco generaciones, en condiciones de laboratorio, utilizando cópula \\ inducida. Los ejemplares fueron expuestos a un fotoperíodo de 12 horas, \\ $24-29^{\circ} \mathrm{C}$ de temperatura y $72-80 \%$ de humedad relativa. Se tomaron \\ medidas de crecimiento y duración del ciclo biológico. La duración de \\ los estados inmaduros fue de 12,6 días manteniéndose uniforme el \\ tamaño promedio larval en las cinco generaciones, mientras que la \\ longevidad obtenida para las hembras y machos fue de 26,0 y 30,8 días \\ respectivamente.
}

\section{INTRODUCCION}

Anopheles (Anopheles) pseudopunctipennis, Theobald 1901, es uno de los vectores más importantes de malaria en las Américas (1); está registrado desde Estados Unidos hasta Chile y Argentina, pasando por las Antillas Menores (2); su amplia distribución determina la transmisión diferencial de malaria, sugiriendo variaciones fisiológicas y morfológicas (3), ya que en la actualidad se reconocen 7 subespecies y/o variedades de esta especie (2); sin embargo, es posible que presente un complejo de especies. La condición de vector de malaria de Anopheles pseudopunctipennis aparentemente varía con la subespecie o variedad, de las citadas por Forattini (1) únicamente Anopheles pseudopunctipennis pseudopunctipennis es considerada de importancia. En Colombia fue encontrado infectado naturalmente con Plasmodium en capturas hechas en Barrancabermeja (Santander), Guacarí (Valle) (4) y en La Unión (Nariño) (5). Los autores, sin embargo, no aclaran la subespecie observada. En el Valle del Cauca se encuentra ampliamente distribuida desde aproximadamente $70 \mathrm{msnm}$ hasta $1350 \mathrm{msnm}(6,7$ y 8); en el valle geográfico de esta región se ha registrado la variedad bifoliata (9).

El ciclo biológico en el campo de Anopheles pseudopunctipennis ha sido estudiado particularmente por Forattini (1) y Gorham y col. (10). Sin embargo, la obtención de datos experimentales ha estado limitada por la ausencia de colonias de producción (11 y 12), lo que puede atribuirse a las dificultades de crianza masal, originadas posiblemente en la condición estenogámica de la especie y sus requerimientos nutricionales; la colonización más exitosa de esta especie (cepa de Panamá) hasta el presente fue mantenida durante 40 generaciones (13). En El Salvador (14) se logró colonizar dos cepas (Ilopango y Huiza) usando cópula inducida.

Dada la abundancia relativa de esta especie en el valle geográfico del río Cauca, la ausencia de estudios

(1) Este artículo corresponde al estudio "Ciclo biológico y establecimiento de una colonia de Anopheles pseudopunctipennis en Cali"; presentado como requisito parcial para optar al título de Biólogo. Investigación financiada por el Programa Especial de las Naciones Unidas, Banco Mundial y la Organización Mundial de la Salud (OMS), para la investigación y entrenamiento en enfermedades tropicales (TDR).

* Departamento de Biología, Facultad de Ciencias, Universidad del Valle, Cali, Colombia.

** Investigador Asociado, Departamento de Microbiología, Facultad de Salud, Universidad del Valle, Cali, Colombia.

***Profesor Asociado, Departamento de Biología, Facultad de Ciencias, Universidad del Valle, Cali, Colombia. 
biológicos de la variedad bifoliata (determinada por comparación de la morfología de la genitalia del macho) así como también la necesidad de tener una colonia experiemental de Anopheles, nos propusimos realizar una crianza masal así como también su ciclo biológico durante cinco generaciones.

\section{MATERIALES Y METODOS}

\section{ESTABLECIMIENTO DE LA COLONIA}

Para iniciar el establecimiento de la colonia con cópula natural, se realizaron capturas de larvas en los lotes experimentales de arroz del Centro Internacional de Agricultura Tropical (CIAT, Palmira) localizado a $1000 \mathrm{msnm}$. Las recolecciones de estos estadios se continuaron una vez por semana de enero a abril de 1984 y se mantuvieron en un laboratorio del Departamento de Microbiología de la Universidad del Valle a una temperatura de $24-29^{\circ} \mathrm{C}$ y una humedad relativa de $72-80 \%$. Al no tener éxito con la cópula natural, se procedió a utilizar cópula inducida, fundamentalmente con el material colectado en el mes de abril.

Las larvas crecieron en bandejas esmaltadas $(38,5 \times 25,5 \times 5,5 \mathrm{~cm})$ con agua traída de los criaderos; tres veces al día se les suministró como alimento galleta para ratón pulverizada más algas (principalmente Spirogira) traídas de los criaderos naturales.

Las bandejas se colocaron en una estantería metálica $(2,1 \times 1,7 \mathrm{~m} \times 50 \mathrm{~cm})$ con un fotoperíodo de doce horas diarias (6 a.m. a 6 p.m.) suministrada por bombillos de $60 \mathrm{w}$, manteniendo la temperatura del agua a $29 \pm 1^{\circ} \mathrm{C} \mathrm{y} \mathrm{pH} 7$. Las pupas producidas se trasladaron a tazas plásticas blancas $(11,3 \times 9,5 \mathrm{~cm})$ con $250 \mathrm{ml}$ de agua de los criaderos y provistas de una tapa de malla en el centro, luego eran pasadas a jaulas metálicas marca Gerberg ( $30 \times 30 \times 30 \mathrm{~cm})$ en donde se dejaban libres los adultos que habían emergido, la taza se retiraba posteriormente. La humedad en la jaula se mantuvo colocando en la parte superior gasa y algodón humedecido. A los adultos se les suministró una solución de miel de abeja al diez por ciento por medio de una bola hecha de gasa y algodón $(5 \mathrm{~cm}$ aproximadamente) la cual se colgó en el centro de la jaula. Las hembras se alimentaron en un comienzo con sangre humana y posteriormente se proporcionó sangre de cobayo.
La cópula inducida se realizó según la técnica descrita en el Manual de prácticas entomológicas (15). Las hembras inseminadas se colocaron en un vial (7 x $2,8 \mathrm{~cm}$ ) previamente preparado con fondo de algodón de $1 \mathrm{~cm}$ de espesor, saturado con agua destilada al igual que un disco de papel de filtro de diámetro del vial que cubre esta capa; el vial fue tapado con una malla de tul, a través de la cual se le suministró alimento y agua a la hembra. Los huevos depositados fueron trasladados a tazas plásticas verdes $(132 \times 48$ $\mathrm{mm}$ ), en cuya base se colocó un círculo de papel de filtro sobresaturado con agua, para permitir su incubación; estas tazas fueron colocadas sobre estanterías metálicas $(2,1 \times 1,7 \mathrm{~m} \times 50 \mathrm{~cm})$ a las cuales se les adaptaron bombillos de $60 \mathrm{w}$ que les proporcionó una intensidad baja de luz.

\section{CICLO BIOLOGICO}

El seguimiento del ciclo de vida se hiŁo a partir de 200 huevos de cinco generaciones (40 por generación), obtenidos del primer ciclo gonotrófico de una hembra por cada generación. Usando un micrómetro ocular a cada huevo se le midió el diámetreo ecuatorial (DE) y el diámetro polar (DP); el porcentaje de eclosión se calculó a partir de 2436 huevos, en las diferentes generaciones. A las larvas obtenidas de los 200 huevos se les practicaron mediciones diarias del ancho de la cápsula cefálica (ACC) y del largo total (LT) hasta completar su ciclo larval; igualmente se midió la duración en días de los estadios larvales, pupa y longevidad de los adultos (desde emergencia hasta muerte).

Los estadios larvales fueron calculados por medio de la regla de Dyar (10) modificando el valor de la constante de crecimiento a 1,5. A los resultados de ancho de cápsula cefálica, largo total, duración del ciclo de desarrollo y longevidad de machos y hembras se les hizo un análisis de varianza (anova 1) para determinar diferencias estadísticamente significativas, entre réplicas de la misma generación y entre las diferentes generaciones, con un nivel de significación de 0,01 $(\propto=0,01)$.

\section{RESULTADOS}

La primera generación de An. pseudopunctipennis se obtuvo a partir de 500 adultos aproximadamente, emergidos de larvas y pupas colectadas en el campo. El número de ejemplares de cada generación aumentó 
progresivamente, hasta la quinta generación cuando fueron suspendidas las observaciones. Al final del experimento se tuvo material vivo de la tercera, cuarta y quinta generación.

\section{CICLO BIOLOGICO}

HUEVOS: El diámetro polar y ecuatorial medido en los 200 huevos de las 5 generaciones varió entre 0,51 y 0,$56 ; 0,12$ y $0,14 \mathrm{~mm}$ respectivamente (tabla 2). El número promedio de huevos por hembra fue de 135,7 , variando entre 122 y 152 huevos en las cinco generaciones (tabla 1). El porcentaje de eclosión medido en 2436 huevos de las cinco generaciones fue de $68,15 \%$ y el período de incubación fue siempre de 2 días (tabla 3).
TABLA 1. Promedio de huevos por hembra en el primer ciclo gonotrófico y su porcentaje de eclosión medido en las cinco generaciones de Anopheles pseudopunctipennis var. bifoliata.

\begin{tabular}{ccc}
\hline GENERACION & $\begin{array}{c}\text { PROMEDIO } \\
\text { HUEVOS/HEMBRA }\end{array}$ & $\begin{array}{c}\text { PORCENTAJE } \\
\text { DEECLOSION }\end{array}$ \\
1 & 139,0 & 74,0 \\
2 & 152,0 & 66,4 \\
3 & 132,0 & 70,4 \\
4 & 133,5 & 66,1 \\
5 & 122,0 & 82,0 \\
Promedio & 135,7 & \\
\hline
\end{tabular}

TABLA 2. Tamaño promedio* de huevo (D.E., D.P.) larva (ancho de cápsula cefálica, largo total) en las cinco generaciones de Anopheles seudopunctipennis var. bifoliata.

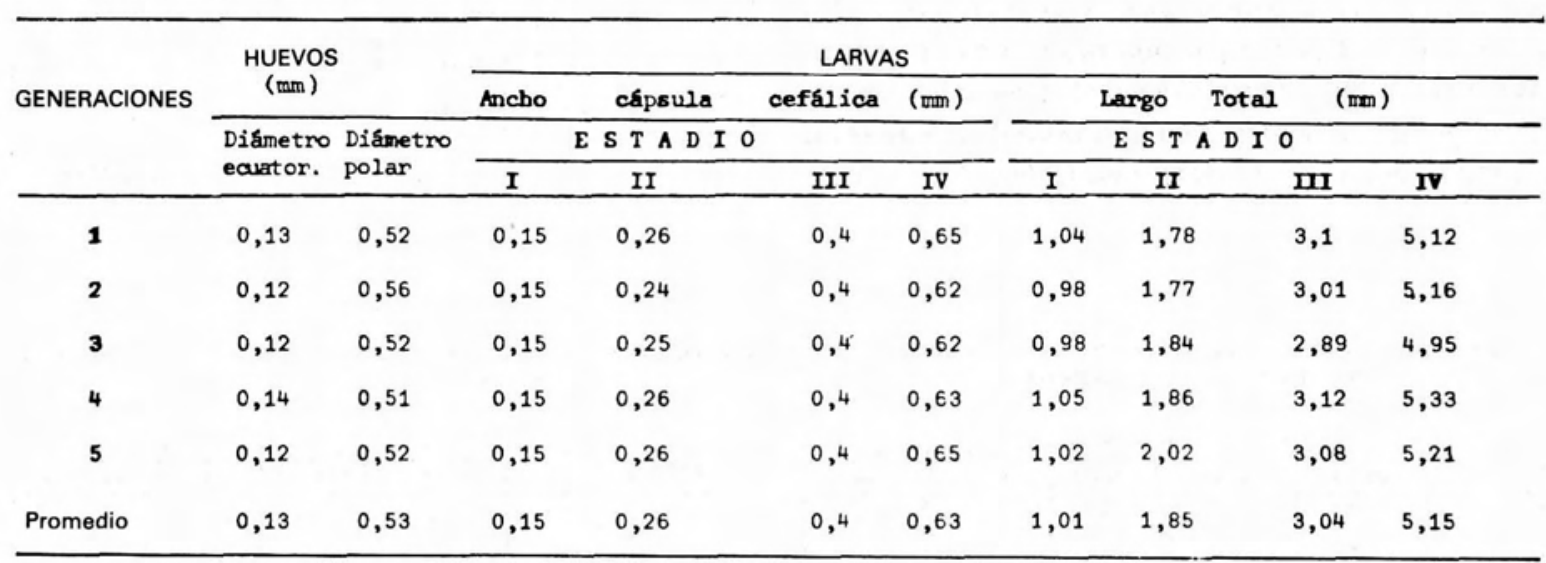

*Promedio de 40 observaciones/generación.

TABLA 3. Duración de huevo, larva, pupa y longevidad en las cinco generaciones de Anopheles seudopunctipennis var. bifoliata.

\begin{tabular}{|c|c|c|c|c|c|c|c|c|c|c|c|}
\hline \multirow{3}{*}{ GENERACIONES } & \multirow{3}{*}{$\begin{array}{l}\text { Duraciôn } \\
\text { Huevo } \\
\text { (horas) }\end{array}$} & \multicolumn{4}{|c|}{ DURACION DE LA LARVA (horas) } & \multirow{3}{*}{$\begin{array}{l}\text { Duración } \\
\text { Total } \\
\text { Larva (L) } \\
\text { (Horas) }\end{array}$} & \multirow{3}{*}{$\begin{array}{l}\text { Duración } \\
\text { Pupa (P) } \\
\text { (Horas) }\end{array}$} & \multirow{3}{*}{$\begin{array}{l}\text { Total } \\
\mathrm{L}+\mathrm{P} \\
\text { (Horas) }\end{array}$} & \multicolumn{2}{|c|}{ DURACION ADULTOS (dias) } & \multirow{3}{*}{$\begin{array}{l}\text { Duración } \\
\text { Total } \\
\text { dias }\end{array}$} \\
\hline & & \multicolumn{4}{|c|}{ ESTADIO } & & & & MACHO & HEMBRA & \\
\hline & & $I$ & 11 & III & IV & & & & & & \\
\hline 1 & 48 & 54 & 43,2 & 41,4 & 61,2 & 199,8 & 48 & 247,8 & 27,78 & 28,92 & 41,02 \\
\hline 2 & 48 & 66,6 & 37,8 & 46,8 & 69 & 220,2 & 48 & 268,2 & 29,83 & 24,54 & 40,93 \\
\hline 3 & 48 & 70,8 & 42 & 44,4 & 67,2 & 224,4 & 48 & 272,4 & 31,86 & 28,02 & 43,7 \\
\hline 4 & 48 & 48 & 34,2 & 41,35 & 63,6 & 189 & 48 & 237 & - & - & - \\
\hline 5 & 48 & 48 & 29,4 & 44,4 & 86,5 & 205,2 & 48 & 253,2 & 33,68 & 23,09 & 40,2 \\
\hline Promedio & 48 & 57,5 & 37,3 & 43,7 & 69,5 & 207,7 & 48 & 255,7 & 30,8 & 26,1 & 41,5 \\
\hline
\end{tabular}


LARVAS: Las larvas como otros culicidos presentaron 4 estadios larvales; datos del tamaño promedio de ancho de cápsula cefálica, largo total y duración total del desarrollo larval de cada uno de los estadios larvales, se presentan en la tabla 2 y 3 respectivamente. El promedio de ancho de cápsula cefálica así como el de largo total no varió significativamente entre bandejas de la misma generación (tabla 4 y 5) ni tampoco entre bandejas de las diferentes generaciones (tabla 7).

El desarrollo larval en las cinco generaciones tuvo una duración de 8,6 días; no se hallaron diferencias significativas entre bandejas de la misma generación (tabla 6) ni entre generaciones (tabla 7).

PUPAS: $\mathrm{Al}$ igual que el período embrionario tuvo una duración promedio de dos días (tabla 3).

ADULTOS: La longevidad promedio de los adultos medida en cuatro generaciones (G1, G2, G3 y G5) fue de 30,8 y 26,0 días para machos y hembras respectivamente (tabla 3 ); resultados de la generación cuatro no se presentan debido a un accidente de laboratorio, haciendo que éstos no sean confiables. No se halló

TABLA 4. ANOVA I Comparación del temeno promedio de oncho de capsula cefalica entre bandejos, en las cinco generación.

\begin{tabular}{|c|c|c|c|c|c|}
\hline GENERACION & \begin{tabular}{|c|} 
FUENTE DE \\
VARIACION
\end{tabular} & \begin{tabular}{c|} 
SUMA DE \\
CUADRADOS
\end{tabular} & $\begin{array}{l}\text { GRADOSDE } \\
\text { LIBERTAD }\end{array}$ & $\begin{array}{l}\text { CUADRADO } \\
\text { MEDIO }\end{array}$ & $\begin{array}{l}\text { RAZON DE } \\
\text { VARIANZA }\end{array}$ \\
\hline \multirow{3}{*}{1} & $\begin{array}{l}\text { ENTRE } \\
\text { GRUPOS }\end{array}$ & $3,4830 \mathrm{E} \cdot 03$ & 3 & $1,1610 \in E-03$ & 0,025 \\
\hline & $\begin{array}{l}\text { DENTRO } \\
\text { GRUPOS }\end{array}$ & 0,5586 & 12 & 0,04654 & \\
\hline & TOTAL & 0,5620 & 15 & & \\
\hline \multirow{3}{*}{2} & $\begin{array}{l}\text { ENTRE } \\
\text { GRUPOS }\end{array}$ & $3,2416 \in-04$ & 3 & $1,080 \quad E-04$ & $2,5085 \varepsilon=08$ \\
\hline & $\begin{array}{l}\text { DENTRO } \\
\text { GRUPOS }\end{array}$ & 0,5169 & 12 & 0,04308 & \\
\hline & TOTAL & 0,5172 & 15 & & \\
\hline \multirow{3}{*}{3} & $\begin{array}{l}\text { ENTRE } \\
\text { GRUPOS }\end{array}$ & $1,2560 \varepsilon \cdot 03$ & 3 & $4,1869 \varepsilon-04$ & 0,01021 \\
\hline & $\begin{array}{l}\text { DENTRO } \\
\text { GRUPOS }\end{array}$ & 0,4918 & 12 & 0,04098 & \\
\hline & TOTAL & 0,4930 & 15 & & \\
\hline \multirow{3}{*}{4} & $\begin{array}{l}\text { ENTRE } \\
\text { GRUPOS }\end{array}$ & $2,4224 E-04$ & 3 & $8,0749 E-0 S$ & I, $8897 \in-03$ \\
\hline & $\begin{array}{l}\text { DENTRO } \\
\text { GRUPOS }\end{array}$ & 0,5128 & 12 & 0,04272 & \\
\hline & TOTAL & 0,5129 & is & & \\
\hline \multirow{3}{*}{5} & $\begin{array}{l}\text { ENTRE } \\
\text { GRUPOS }\end{array}$ & $3,5083 \in-06$ & 3 & $1,1694 \varepsilon=06$ & $1,889 \quad \varepsilon-03$ \\
\hline & $\begin{array}{l}\text { DENTRO } \\
\text { GRUPOS }\end{array}$ & 0,5492 & 12 & 0,0458 & \\
\hline & TOTAL & 0,5492 & 15 & & \\
\hline
\end{tabular}

$0,99^{-5,95}$ diferencia significativa en la longevidad de las hembras y machos en las cuatro generaciones (tabla 8).

\begin{tabular}{|c|c|c|c|c|c|}
\hline GENERACION & \begin{tabular}{|c|} 
FUENTE DE \\
VARIACION \\
\end{tabular} & $\begin{array}{l}\text { SUMA DE } \\
\text { CUADRADOS }\end{array}$ & $\begin{array}{l}\text { GRADOS DE } \\
\text { LIBERTAD }\end{array}$ & \begin{tabular}{|c|} 
CUADRADOO \\
MEDIO \\
\end{tabular} & $\begin{array}{l}\text { RAZON DE } \\
\text { VARIANZA }\end{array}$ \\
\hline \multirow{3}{*}{1} & $\begin{array}{l}\text { ENTRE } \\
\text { GRUPOS }\end{array}$ & 0,2074 & 3 & 0,06912 & 0,02149 \\
\hline & $\begin{array}{l}\text { OENTRO } \\
\text { GRUPOS }\end{array}$ & 38,5846 & 12 & 3,2154 & \\
\hline & TOTAL & 38,7919 & 15 & & \\
\hline \multirow{3}{*}{2} & $\begin{array}{l}\text { ENTRE } \\
\text { GRUPOS }\end{array}$ & 0,0145 & 3 & $4,8430 E-03$ & $1,4521 \in=03$ \\
\hline & $\begin{array}{l}\text { DENTRO } \\
\text { GRUPOS }\end{array}$ & 40,0205 & 12 & 3,3350 & \\
\hline & TOTAL & 40,0381 & 15 & & \\
\hline \multirow{3}{*}{3} & $\begin{array}{l}\text { ENTRE } \\
\text { GRUPOS }\end{array}$ & 0,2778 & 3 & 0,0926 & 0,03122 \\
\hline & $\begin{array}{l}\text { OENTRO } \\
\text { GRUPOS }\end{array}$ & 35,5891 & 12 & 2,9657 & \\
\hline & TOTAL & 35,8669 & 15 & & \\
\hline \multirow{3}{*}{4} & $\begin{array}{l}\text { ENTRE } \\
\text { GRUPOS }\end{array}$ & 0,26258 & 3 & 0,8752 & 0,0250 \\
\hline & $\begin{array}{l}\text { OENTRO } \\
\text { GRUPOS } \\
\end{array}$ & 41,9252 & 12 & 3,4938 & \\
\hline & TOTAL & 42,1879 & 15 & & \\
\hline \multirow{3}{*}{5} & $\begin{array}{l}\text { ENTRE } \\
\text { GRUPOS }\end{array}$ & 0,0195 & 3 & $6,5099 E-03$ & $2,0423 E-03$ \\
\hline & $\begin{array}{l}\text { OENTRO } \\
\text { GRUPOS }\end{array}$ & 38,2498 & 12 & 3,1875 & \\
\hline & TOTAL & 38,2694 & 15 & & \\
\hline
\end{tabular}

TABLA 6. ANOVA 1 Comparación entre bondejas dentro de cada uno de los generaciones, en cuanto a su duración larval.

\begin{tabular}{|c|c|c|c|c|c|}
\hline GENERACION & $\begin{array}{c}\text { FUENTE OE } \\
\text { VARIACION } \\
\end{array}$ & $\begin{array}{c}\text { SUMA DE } \\
\text { CUADRADOS } \\
\end{array}$ & $\begin{array}{l}\text { GRADOS DE } \\
\text { LIBERTAD }\end{array}$ & $\begin{array}{l}\text { CUADRADO } \\
\text { MEDIO }\end{array}$ & \begin{tabular}{|l} 
RAZON DE \\
VARIANZA \\
\end{tabular} \\
\hline \multirow{3}{*}{1} & $\begin{array}{l}\text { ENTRE } \\
\text { GRUPOS }\end{array}$ & 35,64 & 3 & 11,88 & 0,07231 \\
\hline & $\begin{array}{l}\text { OENTRO } \\
\text { GRUPOS }\end{array}$ & 1971,4 & 12 & 164,28 & \\
\hline & TOTAL & 2006,9 & 15 & & \\
\hline \multirow{3}{*}{2} & $\begin{array}{l}\text { ENTRE } \\
\text { GRUPOS }\end{array}$ & 73,08 & 3 & 24,36 & 0,09026 \\
\hline & $\begin{array}{l}\text { DENTRO } \\
\text { ORUPOS }\end{array}$ & 3238,5 & 12 & 269,80 & \\
\hline & TOTAL & 3311,6 & 15 & & \\
\hline \multirow{3}{*}{3} & $\begin{array}{l}\text { ENTRE } \\
\text { GRUPOS }\end{array}$ & 289,44 & 3 & 96,48 & 0,2533 \\
\hline & $\begin{array}{l}\text { DENTRO } \\
\text { GRUPOS }\end{array}$ & 4570,6 & 12 & 380,9 & \\
\hline & TOTAL & 4859,9 & 15 & & \\
\hline \multirow{3}{*}{4} & $\begin{array}{l}\text { ENTRE } \\
\text { ORUPOS }\end{array}$ & 77,76 & 3 & 25,92 & 0,1369 \\
\hline & $\begin{array}{l}\text { DENTRO } \\
\text { GRUPOS }\end{array}$ & 2272,3 & 12 & 189,36 & \\
\hline & TOTAL & 2350,1 & 15 & & \\
\hline \multirow{3}{*}{5} & $\begin{array}{l}\text { ENTRE } \\
\text { GRUPOS }\end{array}$ & 25,71 & 3 & 8,57 & 0,01416 \\
\hline & $\begin{array}{l}\text { DENTRO } \\
\text { GRUPOS }\end{array}$ & 7263,9 & 12 & 605,33 & \\
\hline & TOTAL & 7289,67 & 15 & & \\
\hline
\end{tabular}


TABLA 7. ANOVA 1 Comparación del tameno promedio de ancho de capsula ce falico del lorgo total entre los cinco generaciones.

\begin{tabular}{|c|c|c|c|c|c|}
\hline $\begin{array}{l}\text { TIPO DE } \\
\text { COMPARACION }\end{array}$ & $\begin{array}{l}\text { FUENTE DE } \\
\text { VARIACION }\end{array}$ & $\begin{array}{l}\text { SUMA DE } \\
\text { CUADRADOS }\end{array}$ & $\begin{array}{l}\text { GRADOS DE } \\
\text { LIBERTAD }\end{array}$ & $\begin{array}{l}\text { CUADRADO } \\
\text { MEDIO }\end{array}$ & $\begin{array}{l}\text { RAZON DE } \\
\text { VARIANZA }\end{array}$ \\
\hline \multirow{3}{*}{$\begin{array}{l}\text { ANCHO DE } \\
\text { CAPSULAA } \\
\text { CEFALICA }\end{array}$} & $\begin{array}{l}\text { ENTAE } \\
\text { GRUPOS }\end{array}$ & $1,099 \quad E-03$ & 4 & $2,740 \quad E-04$ & 3,106 \\
\hline & $\begin{array}{l}\text { DENTRO } \\
\text { GRUPOS }\end{array}$ & $1,327 \quad E-03$ & 13 & $0,040 \mathrm{E}-0 \mathrm{~S}$ & \\
\hline & TOTAL & $2,427 \quad E-03$ & 19 & & \\
\hline \multirow{3}{*}{$\begin{array}{l}\text { LARGO } \\
\text { TOTALL }\end{array}$} & $\begin{array}{l}\text { ENTRE } \\
\text { GRUPOS }\end{array}$ & 0,0953 & 4 & 0,0238 & 1,8025 \\
\hline & $\begin{array}{l}\text { OENTRO } \\
\text { GRUPOS }\end{array}$ & 0,19832 & 15 & 0,0132 & \\
\hline & TOTAL & 0,2936 & 19 & & \\
\hline
\end{tabular}

TABLA 8. ANOVA 1 Comparación de la longevidad de los machos y de las hembras en 4 generaciones.

\begin{tabular}{|c|c|c|c|c|c|}
\hline $\begin{array}{l}\text { TIPO DE } \\
\text { COMPARACION }\end{array}$ & $\begin{array}{l}\text { FUENTE DE } \\
\text { VARIACION }\end{array}$ & \begin{tabular}{|} 
SUMA DE \\
CUADRADOS
\end{tabular} & $\begin{array}{l}\text { GRADOS DE } \\
\text { LIBERTAD }\end{array}$ & $\begin{array}{l}\text { CUADRADO } \\
\text { MEDIO }\end{array}$ & $\begin{array}{l}\text { RAZON DE } \\
\text { VARIANZA }\end{array}$ \\
\hline \multirow{3}{*}{$\begin{array}{l}\text { LONGEVIDAO } \\
\text { DE LOS } \\
\text { MACHOS }\end{array}$} & $\begin{array}{l}\text { ENTRE } \\
\text { ORUPOS }\end{array}$ & 77,93 & 3 & 25,98 & 1,717 \\
\hline & $\begin{array}{l}\text { DENTRO } \\
\text { GRUPOS }\end{array}$ & 181,54 & 12 & 15,13 & \\
\hline & TOTAL & 259,5 & 15 & & \\
\hline \multirow{3}{*}{$\begin{array}{l}\text { LONGEVIDAO } \\
\text { OE LAS } \\
\text { HEMERAS }\end{array}$} & $\begin{array}{l}\text { ENTRE } \\
\text { GRUPOS }\end{array}$ & 92,52 & 3 & 30,84 & 0,5728 \\
\hline & $\begin{array}{l}\text { DENTRO } \\
\text { GRUPOS }\end{array}$ & 646,08 & 12 & 53,84 & \\
\hline & TOTAL & 738,6 & 15 & & \\
\hline
\end{tabular}

\section{DISCUSION}

Es bien conocida la dificultad que existe para establecer colonias de anofelinos. La colonización más exitosa de Anopheles pseudopunctipennis, fue mantenida durante 40 generaciones de una cepa de Panamá sin cópula inducida (13).

La principal limitación para el establecimiento de la colonia estuvo en que esta especie no copuló naturalmente coincidiendo con los registros de Darsie y López (14) y Warren y col. (17) debido posiblemente a la condición estenogámica de la especie ya que posiblemente la densidad de la población manejada experimentalmente no fue suficiente para la formación de enjambre por parte de los machos; por lo tanto la colonia se mantuvo utilizando cópula inducida.

El porcentaje de eclosión $(68,15 \%)$ fue superior al registrado para las colonias de Ilopango (57\%) y Huiza (56\%) en El Salvador (14). El número de huevos por hembra fue menor que el obtenido por Baerg (13) (menos de 200 y algunas veces más de 300 ), las diferencias probablemente se deben a dificultades por manipulación en la cópula inducida, tiempo de insemina- ción insuficiente, diferencias en la temperatura y humedad relativa o características intrínsecas de la variedad.

El período de incubación de los huevos y el désarrollo de la pupa coincide parcialmente con el registro de duración de 2 a 3 días $\left(22^{\circ} \mathrm{C}-29^{\circ} \mathrm{C} ; 60-80 \mathrm{HR}\right)$ (13). El promedio de la duración larval fue mayor (8,6 días) comparado con el obtenido de 8 días por Baerg (13).

Las diferencias halladas en el período de incubación en la duración larval y en el desarrollo de la pupa son debidas posiblemente a que el rango de temperatura $\mathrm{y}$ humedad relativa nuestro fue menor, a que se traten de sub-especies y/o variedades diferentes, a las condiciones alimenticias u otros factores no considerados.

La longevidad hallada para los adultos fue de 30,8 y 26,0 días para los machos y hembras respectivamente, observándose un comienzo de adaptación dado por el incremento progresivo de la duración de los machos. Baerg (13) registró un promedio de supervivencia de 20,0 y 25,0 días para machos y hembras respectivamente. La longevidad para las hembras fue menor con respecto a los machos, no coincidiendo con lo registrado por Baerg (13), quien obtuvo mayor longevidad para las hembras; posiblemente estas diferencias se dan por la dificultad que presentaron para ser alimentadas y por la incidencia del éter utilizado como anestésico para la cópula inducida.

Los resultados presentados muestran que usando la técnica de cópula inducida es posible mantener $A n$. pseudopunctipennis variedad bifoliata bajo condiciones de laboratorio en volumen adecuado para realizar trabajos de investigación tales como susceptibilidad al parásito, ciclo gonotrófico, dietas alimenticias, citogenética y resistencia a insecticidas.

\section{SUMMARY}

Anopheles pseudopunctipennis, variety bifoliata, was colonized for five generations under laboratory conditions, using induced copulation. The specimens were placed on a 12 hour photoperiod, at $24-29^{\circ} \mathrm{C}$ of temperature and $72-80 \%$ of relative humidity. Data were collected for growth and life cycle. The duration of the immature stages was 12.6 days; a standard larval size was osberved through the five generations period. The longevity of female and male was 26.0 and 30.8 days respectivety. 


\section{AGRADECIMIENTOS}

Agradecemos al Dr. Alberto Alzate, Jefe del Departamento de Microbiología de la Universidad del Valle, por su colaboración y orientación en la parte estadística. A los doctores María del Pilar Carrillo de Olano, Victor Olano y Ricardo Restrepo por su valiosa ayuda en el entrenamiento de manejo y colonización de anofelinos y al Centro Internacional de Agricultura Tropical (CIAT), al brindar su colaboración en la obtención de material de campo.

\section{BIBLIOGRAFIA}

1. Forattini OP. Entomología Médica. Sao Paulo: Facultade de Higiene e Saúde Pública 1962: 309-311.

2. Knight K, Stone A. A catalog of the mosquitoes of the world. The Thomas Say Foundation 1977; $6: 27$.

3. Baker RH, Kitzmiller JB, Chowdaiah BN. Salivary gland chromosomes of Anopheles pseudopunctipennis pseudopunctipennis. Bull WHO 1965; 33:837.

4. Cadena MA. Disección de mosquitos Anopheles (resumen de resultados). Rev Fac Med Bogotá 1938; 7:328.

5. Rey H. Conocimiento actual sobre vectores en Colombia. An Soc Biol Bogotá 1950; 4:85.

6. Renjifo S. Notas entomológicas regionales. (Tesis) Bogotá, Colombia: Universidad Nacional de Colombia, 1944.
7. Fajardo P. Especies de Anopheles.(Tesis) Universidad del Valle, Cali. 1983; 116

8. Muñoz LC, y R Parra. (Tesis) Universidad del Valle, Cali. $1984 ; 134$.

9. Osorno E, y F Muñoz. Una nueva variedad de Anopheles pseudopunctipennis. Caldasia. 1948; 5:105.

10. Gorham JR, CJ, Stojanovich HG Scott. Clave ilustrada para los mosquitos anofelinos de Sudamérica Occidental Mosq. syst. 1973; 5:99.

11. Trembley HL. Mosquito culture techniques and experimental procedures. Amer Mosq Cont Assoc Bull. 1955; 3:73.

12. Gerberg EJ. Manual for mosquito rearing and experimental techniques. Amer Mosq Cont Assoc Bull 1970; 5:107.

13. Baerg DC. Colonization of Anopheles pseudopunctipennis in Panamá. J Med Entomol 1971; 8:180.

14. Darsie RF, GA López. Studies of colonization of El Salvador strains of Anopheles pseudopunctipennis pseudopunctipennis. Mosq. News 1980; 40:194.

15. WORLD HEALTH ORGANIZATION. Manual on practical entomology in malaria, Part III. Methods and Techniques. World Health Organization, Geneva. 1975; 175-179.

16. Wigglesworth VB. The principles of insect physiology. 6 ed. Methuen and Co Ltd EC-4. 1967; 54.

17. Warren M, WE Collins GM, Jeffery BB Richardson. Anopheles pseudopunctipennis : Laboratory maintenance and malaria susceptibility of a strain from El Salvador. Am J Trop Med Hyg 1980; 4:503. 